\title{
The Turing Model for Biological Pattern Formation
}

\author{
Philip K. Maini and Thomas E. Woolley
}

\begin{abstract}
How spatial patterning arises in biological systems is still an unresolved mystery. Here, we consider the first model for spatial pattern formation, proposed by Alan Turing, which showed that structure could emerge from processes that, in themselves, are non-patterning. He therefore went against the reductionist approach, arguing that biological function arises from the integration of processes, rather than being attributed to a single, unique, process. While still controversial, some 65 years on, his model still inspires mathematical and experimental advances.
\end{abstract}

\section{Biological Pattern Formation}

Biological systems exhibit a diverse range of patterns, such as animal pigmentation patterns, limb skeletal structures, etc. (Fig. 1). Despite decades of research, a detailed understanding of how these patterns arise still eludes us. We know many of the genes involved and can map out the spatiotemporal dynamics of some of them, but how these dynamics arise is still largely a mystery. In 1952, the logician, computer scientist, code breaker and mathematician Alan Turing proposed a novel mathematical model for pattern formation [1]. He hypothesised that the patterns we see arise due to cells responding to underlying pre-patterns of chemical concentrations. He termed these chemicals morphogens, and showed that spatially heterogeneous patterns could arise in systems in which these chemicals reacted with each

Philip K. Maini

Wolfson Centre for Mathematical Biology, Mathematical Institute, University of Oxford, Andrew Wiles Building, Radcliffe Observatory Quarter, Woodstock Road, Oxford OX2 6GG, UK e-mail: maini@maths.ox.ac.uk

Thomas E. Woolley

Wolfson Centre for Mathematical Biology, Mathematical Institute, University of Oxford, Andrew Wiles Building, Radcliffe Observatory Quarter, Woodstock Road, Oxford OX2 6GG, UK e-mail: woolley@maths.ox.ac.uk 
other and also underwent diffusion - a phenomenon termed diffusion-driven instability. Making the further assumption that cell fate was determined in a morphogen concentration-dependent manner, the chemical pre-pattern would manifest itself in a pattern composed of spatially heterogeneous cell fates. In Sect. 2 we will describe the phenomenon of diffusion-driven instability and deduce the properties exhibited by the resultant patterns. We will also give some examples of reaction-diffusion systems. In Sect. 3 we present some applications and in Sect. 4 we will present conclusions and discussion.

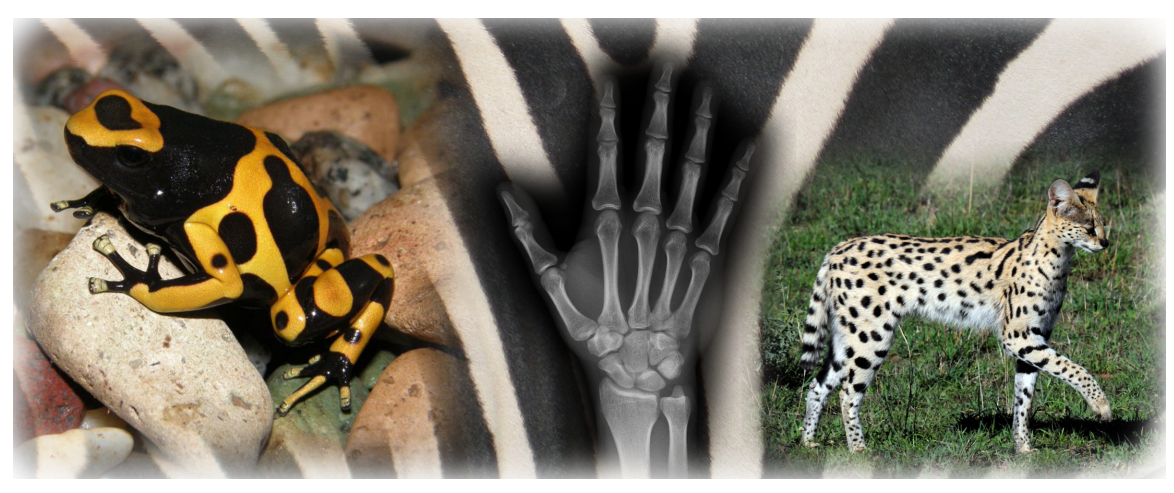

Fig. 1 Examples of biological pattern formation. Zebra stripes are shown in the background and going from left to right: poison arrow frog labyrinthine pigmentation pattern; digit pattern of a human; serval spots transitioning to stripes on the tail.

\section{Mathematical Model}

\subsection{Diffusion is stabilising}

Let us consider the case of a chemical, concentration $u(x, t)$, diffusing in space $x$ (assumed to be in one dimension for simplicity), where $t$ is time. Let us also assume that the chemical is being produced at a rate $f(u)$ where $f$ is typically either a polynomial, or rational, function of $u$. Then

$$
\frac{\partial u}{\partial t}=D \frac{\partial^{2} u}{\partial x^{2}}+f(u)
$$

where $D>0$ is the diffusion coefficient (assumed constant), is the reaction-diffusion equation satisfied by $u(x, t)$.

We will assume further that the spatial domain is $[0, L]$ for some $L>0$ and that 
the chemical concentration at the edge of the domain is fixed at some value $u_{0}$, that is,

$$
u(x, t)=u_{0} \text { at } x=0, L \text { and } \forall t .
$$

This is often called a Dirichlet boundary condition. Furthermore, suppose that $f\left(u_{0}\right)=0$. Then, $u(x, t)=u_{0}$ satisfies equation (1) and the boundary conditions (2) and is termed a spatially uniform steady state for $u$. To find the linear stability of this state we wish to determine if a small perturbation $\hat{u}(x, t)$ from the steady state will grow or decay in time. Substituting into equation (1), expanding $f$ in a Taylor series and keeping only the linear terms, we have:

$$
\frac{\partial \hat{u}}{\partial t}=D \frac{\partial^{2} \hat{u}}{\partial x^{2}}+f^{\prime}\left(u_{0}\right) \hat{u}
$$

where $f^{\prime}=d f / d u$ and we have used the fact that $f\left(u_{0}\right)=0$. Furthermore, $\hat{u}$ satisfies $\hat{u}(x, t)=0$ at $x=0, L$.

In the case where $D=0$, equation (3) has the solution

$$
\hat{u}(x, t)=\hat{u}_{0} \exp \left(f^{\prime}\left(x_{0}\right) t\right)
$$

where $\hat{u}_{0}$ is the initial perturbation. Clearly, if $f^{\prime}\left(u_{0}\right)<0$ then the steady state is linearly stable (as $t$ tends to infinity, $\hat{u}(t)$ tends to zero) while if $f^{\prime}\left(u_{0}\right)>0$, then the perturbation grows and the steady state is linearly unstable.

Now suppose that $D>0$ in (3). Then, using the method of separation of variables, and taking into account the boundary conditions (2), the solution for $\hat{u}(t)$ is the Fourier sine series

$$
\hat{u}(x, t)=\sum_{n=1}^{n=\infty} a_{n} \sin \left(\frac{n \pi x}{L}\right) \exp \left(\lambda_{n} t\right)
$$

where $\lambda_{n}=f^{\prime}\left(u_{0}\right)-D(n \pi / L)^{2}$, for $n=1,2, \ldots$, and $a_{n}$ are determined by equating the solution to the Fourier sine series of the initial condition for the perturbation. Now we see that even when $f^{\prime}\left(u_{0}\right)>0$, if $D>f^{\prime}\left(u_{0}\right)(L / \pi)^{2}$, it follows that $\lambda_{n}<0 \forall n$, that is, each term $\sin (n \pi x / L)$ in the Fourier expansion, termed an admissible mode, will have either an exponentially decaying amplitude (if $a_{n} \neq 0$ ) or zero amplitude (if $a_{n}=0$ ) and so $\hat{u}(x, t)$ tends to zero as $t$ tends to infinity. Therefore, the steady state $u=u_{0}$, although unstable in the absence of diffusion, is stabilised by the presence of diffusion. Hence, diffusion is stabilising.

Note that if the boundary conditions were instead zero flux (so-called homogeneous Neumann) boundary conditions $(\partial u / \partial x=0$ at $x=0, L \forall t)$ then the solution to the linearised system would be a Fourier cosine series and so, in the presence of diffusion, the zeroth mode (constant) term in the Fourier expansion could still grow but every spatially heterogeneous (patterned) term, $\cos (n \pi x / L)$, would have negative growth rate for sufficiently large $D$. For periodic boundary conditions $(u(0, t)=u(L, t) \forall t)$, the Fourier series solution would now be a combination of sines and cosines but the above arguments still hold. 


\subsection{Diffusion is de-stabilising}

The previous mathematical result, namely that diffusion is a stabilising process, also agrees with our intuition, for example if we think of heat. The genius of Turing was to show that this was not necessarily the case if there was more that one chemical, that is, if we had a reaction-diffusion system. Let $u(x, t)$ and $v(x, t)$ be two chemicals satisfying the equations:

$$
\frac{\partial u}{\partial t}=D_{1} \frac{\partial^{2} u}{\partial x^{2}}+f(u, v), \quad \frac{\partial v}{\partial t}=D_{2} \frac{\partial^{2} v}{\partial x^{2}}+g(u, v),
$$

where $f(u, v)$ and $g(u, v)$ are functions describing the reaction kinetics of the morphogens represented by $u$ and $v$, and $D_{1}$ and $D_{2}$ are constant (positive) diffusion coefficients. For simplicity, let us assume that once again $x$ is the finite domain $[0, L]$ and that $u$ and $v$ satisfy homogeneous Neumann boundary conditions.

Now suppose there there are positive values $\left(u_{0}, v_{0}\right)$ such that $f\left(u_{0}, v_{0}\right)=g\left(u_{0}, v_{0}\right)=$ 0 . Then $\left(u_{0}, v_{0}\right)$ is spatially uniform steady state of the system (6). To examine the linear stability of this steady state we extend the analysis in Sect. 2.1 by deriving equations for small perturbations $(\hat{u}(x, t), \hat{v}(x, t))$ to the steady state. Substituting into equations (6), expanding $f$ and $g$ in Taylor series and recalling that $f\left(u_{0}, v_{0}\right)=g\left(u_{0}, v_{0}\right)=0$, we arrive (ignoring higher order terms) at the linearised system:

$$
\frac{\partial \hat{u}}{\partial t}=D_{1} \frac{\partial^{2} \hat{u}}{\partial x^{2}}+f_{u} \hat{u}+f_{v} \hat{v}, \quad \frac{\partial \hat{v}}{\partial t}=D_{2} \frac{\partial^{2} \hat{v}}{\partial x^{2}}+g_{u} \hat{u}+g_{v} \hat{v},
$$

where $f_{u}, f_{v}, g_{u}, g_{v}$ denote the partial derivatives of $f$ and $g$ evaluated at the steady state $\left(u_{0}, v_{0}\right)$. We may re-write this in the more concise form:

$$
\frac{\partial \hat{\boldsymbol{u}}}{\partial t}=\boldsymbol{D} \frac{\partial^{2} \hat{\boldsymbol{u}}}{\partial x^{2}}+\boldsymbol{J}(\hat{\boldsymbol{u}})
$$

where

$$
\hat{\boldsymbol{u}}=\left(\begin{array}{c}
\hat{u}(x, t) \\
\hat{v}(x, t)
\end{array}\right), \boldsymbol{D}=\left(\begin{array}{cc}
D_{1} & 0 \\
0 & D_{2}
\end{array}\right) \text { and } \boldsymbol{J}=\left(\begin{array}{ll}
f_{u} & f_{v} \\
g_{u} & g_{v}
\end{array}\right) \text {. }
$$

We generalise the analysis in Sect. 2.1 by looking for a solution of the form $\hat{\boldsymbol{u}}(x, t)=\boldsymbol{a} \exp \left(i k x+\lambda\left(k^{2}\right) t\right)$ where, again, we are looking for a separable solution, in this case with $\boldsymbol{a}$ a constant vector and the $x$ component of the solution is written as $\exp (i k x)-$ a convenient way to encompass the Fourier components. Substituting this into equation (8) we arrive at the equation

$$
\left(\boldsymbol{J}-\boldsymbol{D} k^{2}-\lambda \boldsymbol{I}\right) \boldsymbol{a}=\mathbf{0},
$$

where $I$ is the $2 \times 2$ unit matrix. For non-trivial solutions, we thus require that the matrix multiplying the vector $\boldsymbol{a}$ is singular, that is,

$$
\operatorname{Det}\left(\boldsymbol{J}-\boldsymbol{D} k^{2}-\lambda \boldsymbol{I}\right)=0
$$


where Det denotes the determinant. This is an eigenvalue problem, that is, the temporal growth rate, $\lambda$, is the eigenvalue of the matrix $\boldsymbol{J}-\boldsymbol{D} k^{2}$ and is, in fact, a function of the wave number, $k$.

Now, in the previous subsection, we showed that in the case of a single reactiondiffusion equation, a spatially uniform steady state, linearly stable in the absence of diffusion, could be stabilised in the present of diffusion. Here, Turing showed the opposite. Let us consider the case when $D_{1}=D_{2}=0$. Then $\lambda$ is simply the eigenvalue of the matrix $\boldsymbol{J}$ and satisfies the eigenvalue problem

$$
\lambda^{2}-\left(f_{u}+g_{v}\right) \lambda+\left(f_{u} g_{v}-f_{v} g_{u}\right)=0 .
$$

For the spatially uniform steady state to be stable we require both solutions to the eigenvalue equation (12) to have negative real part, and this will be true if the following two conditions hold:

$$
f_{u}+g_{v}<0, \text { and } f_{u} g_{v}-f_{v} g_{u}>0 .
$$

Now, in the presence of diffusion ( $D_{1}$ and $D_{2}$ both non-zero), the eiqenvalue problem, from equation (11), relating the growth rate, $\lambda$, to the wave number, $k$, is

$$
\lambda^{2}-b\left(k^{2}\right) \lambda+c\left(k^{2}\right)=0,
$$

where

$b\left(k^{2}\right)=f_{u}+g_{v}-\left(D_{1}+D_{2}\right) k^{2}$ and $c\left(k^{2}\right)=D_{1} D_{2} k^{4}-\left(D_{2} f_{u}+D_{1} g_{v}\right) k^{2}+f_{u} g_{v}-f_{v} g_{u}$.

In this case, we wish diffusion to be de-stabilising and a necessary condition for this to hold true is that at least one of the roots, $\lambda\left(k^{2}\right)$, of equation (14) must have a positive real part for some non-zero (positive) $k^{2}$. This can happen if either $b\left(k^{2}\right)>0$ or $c\left(k^{2}\right)<0$. However the first condition in (13), and the fact that the diffusion coefficients are non-negative, ensures that $b\left(k^{2}\right)<0$, so we require $c\left(k^{2}\right)<0$. For this to occur, the second condition in (13) forces $D_{2} f_{u}+D_{1} g_{v}$ to be positive as a necessary condition. More precisely, we require

$$
D_{2} f_{u}+D_{1} g_{v}>2 \sqrt{D_{1} D_{2}\left(f_{u} g_{v}-f_{v} g_{u}\right)}>0 .
$$

Conditions (13) and (16) ensure that the uniform steady state is linearly stable in the absence of diffusion but has at least one $k$ for which $\lambda\left(k^{2}\right)$ has positive real part. However, to satisfy the zero flux boundary conditions, admissible modes are restricted to $k=n \pi / L$ for at least one integer value $n$. This leads to the 4 th condition:

$$
k_{-}^{2}<\left(\frac{n \pi}{L}\right)^{2}<k_{+}^{2} \text { where } k_{ \pm}^{2}=\frac{f_{u}+g_{v} \pm \sqrt{\left(f_{u}+g_{v}\right)^{2}-4 D_{1} D_{2}\left(f_{u} g_{v}-f_{v} g_{u}\right)}}{2 D_{1} D_{2}} .
$$

Hence, if these conditions are satisfied for at least one integer value, $n>0$, we 
see that a spatially uniform steady state, stable in the absence of diffusion, becomes unstable in the presence of diffusion. This is termed diffusion-driven instability and is an example of self-organisation (or sometimes termed an emergent phenomenon).

A number of key properties are immediately apparent from these conditions:

1. The diffusion coefficients must be unequal. This follows from the first inequality in (13) and (16), because if $D_{1}=D_{2}=D>0$ then we can divide inequality (16) by $D$ to obtain $f_{u}+g_{v}>0$, which contradicts the first inequality of (13).

2. The matrix of partial derivatives $\boldsymbol{J}$ must take one of the following two forms:

$$
\boldsymbol{J}_{\boldsymbol{p}}=\left(\begin{array}{c}
+- \\
+-
\end{array}\right) \text { or } \boldsymbol{J}_{\boldsymbol{c}}=\left(\begin{array}{c}
-- \\
++
\end{array}\right) .
$$

This follows from the first inequality in (13) and (16) and observing that the matrix forms

$$
\boldsymbol{J}_{\boldsymbol{p}}=\left(\begin{array}{c}
-+ \\
-+
\end{array}\right) \text { and } \boldsymbol{J}_{\boldsymbol{c}}=\left(\begin{array}{c}
++ \\
--
\end{array}\right)
$$

are in fact captured, respectively, by $\boldsymbol{J}_{\boldsymbol{p}}$ and $\boldsymbol{J}_{\boldsymbol{c}}$, by appropriately re-defining $u$ and $v$ or $f$ and $g$. In detail, from the first inequality in (13) and (16) it follows that $f_{u}$ and $g_{v}$ must have opposite signs. Hence $f_{u} g_{v}<0$, and the second inequality of (13) forces $f_{v} g_{u}$ to be less than zero, implying that $f_{v}$ and $g_{u}$ have opposite signs.

3. Minimum domain size for pattern formation. This follows from inequality (17). For fixed parameter values in the reaction-diffusion model, for $L$ sufficiently small, this inequality cannot be satisfied for non-zero $n$.

4. As the domain size increases, the pattern becomes more complicated for two reasons: (i) the lower inequality of equation (17) means that the minimum allowable wave mode increases. Explicitly, the pattern appearing on a larger domain will have a smaller wavelength (i.e. the peaks will be packed closer together), when compared to the pattern on a smaller domain; (ii) the range of allowable modes increases. This again follows from inequality (17) by observing that as $L$ increases the number if viable integers must also increase.

5. The idea from point 4 can be extended to higher dimensions. For example, if the spatial domain is the 2-dimensional rectangle $\left[0, L_{x}\right] \times\left[0, L_{y}\right]$ then the admissible modes take the form (for zero flux boundary conditions)

$$
\cos \left(n \pi x / L_{x}\right) \cos \left(m \pi y / L_{y}\right),
$$

where $k^{2}=\left(n \pi / L_{x}\right)^{2}+\left(m \pi / L_{y}\right)^{2}$ and $n=0,1,2, \ldots, m=0,1,2, \ldots$ Clearly, if $L_{y}$ is very small, while $L_{x}$ is large (that is, the domain is long and thin), then from the obvious extension of condition (17) to this case, it follows that $m=0$ and so any spatially patterned structure will vary only in the $x$-direction, that is, the system will exhibit stripes. However, if $L_{x}$ and $L_{y}$ are both large, then (17) can hold for $n$ and $m$ both non-zero. In this case we have spots. Note that in 2 dimensions we have the issue of degeneracy. For example, suppose $L_{x}=L_{y}=1$ and $\left(u_{0}, v_{0}\right)$ was unstable to a mode with $k^{2}=25 \pi^{2}$, then the admissible modes would have wave number pairs $(5,0),(0,5)$ (both corresponding to stripes) and 
$(3,4),(4,3)$ (both corresponding to spots). In this case, initial conditions and the form of the non-linear terms determine which mode is selected (or indeed the solution could be a combination of all possibilities). More recently, these results have been generalised using an energy function to show that pattern selection can be determined by investigating the stationary solutions of an associated FokkerPlanck equation [2].

It is important to point out that the above analysis, and properties derived, hold for linear theory, while the original system is non-linear. The obvious question to ask is, do the linear results hold for the non-linear system? While this can be answered to some extent by carrying out a weakly non-linear analysis in the vicinity of a primary bifurcation point $[3,4]$, we need to resort to numerical solution of the non-linear system for a fuller answer. In Fig. 2 we show some results of numerical simulations of the full non-linear system (see Sect. 2.3) to illustrate the properties 1-4.

In the above, if the linearised kinetics are represented at $\left(u_{0}, v_{0}\right)$ by $\boldsymbol{J}_{\boldsymbol{p}}$ the system is termed a pure activator-inhibitor system while, if they are represented by $\boldsymbol{J}_{\boldsymbol{c}}$ the system is called a cross activator-inhibitor system or a substrate-depletion system. We now explain this terminology. For the case $\boldsymbol{J}_{\boldsymbol{p}}$ we see that at the spatially uniform steady state, $f_{u}>0$ and $f_{v}<0$. Hence, at steady state, $u$ is activating its own production, but $v$ is inhibiting the production of $u$. Moreover, $g_{u}>0$ and $g_{v}<0$ which means that $u$ activates the production of $v$. Hence, $u$ is termed an activator and $v$ is termed an inhibitor. Note further that from the first inequality in (13), and (16) it follows that $D_{2}>D_{1}$. That is, the inhibitor diffuses more widely than the activator. This leads to the self-organising patterning principle of short-range activation long-range inhibition [5]. For the case $\boldsymbol{J}_{c}, u$ is a substrate that produces $v$ but is itself depleted. Note that if we calculate the eigenvector $\boldsymbol{a}$ in the case of $\boldsymbol{J}_{\boldsymbol{p}}$ then equating the second component to zero in the vector equation (8) forces the components of $u$ and $v$ to have the same sign, that is, the solutions are in phase. Conversely, for $\boldsymbol{J}_{\boldsymbol{c}}$, equating the first component to zero in the vector equation (8) implies that $u$ and $v$ must have opposite signs and therefore the solutions are 180 degrees out of phase (see 2(a), (b)).

\subsection{Defining the reaction kinetics}

The functions $f(u, v)$ and $g(u, v)$ can take many forms, too numerous for us to list them all, so we simply give a small sample here. Perhaps the best known is the Gierer-Meinhardt model variant [5] that, when non-dimensionalised, takes the form

$$
f(u, v)=a-b u+\frac{u^{2}}{v} ; g(u, v)=u^{2}-v,
$$

where $a$ and $b$ are positive constants. Here, the model has been constructed such that $v$ inhibits $u$ but $u$ activates $v$. Another model of this class presented by Gierer and 


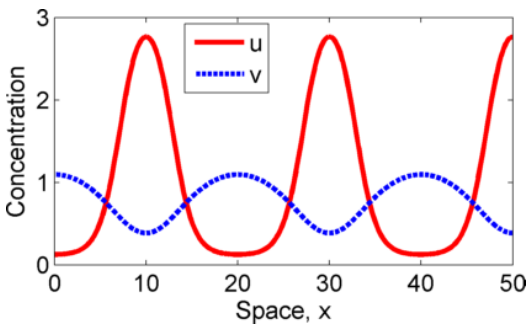

(a)

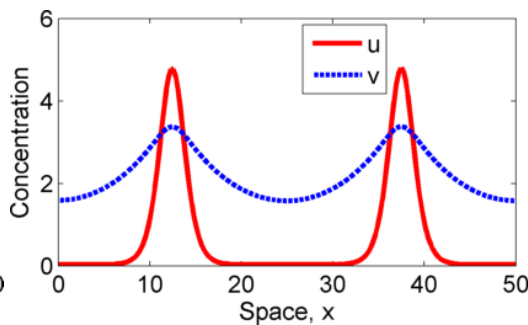

(b)
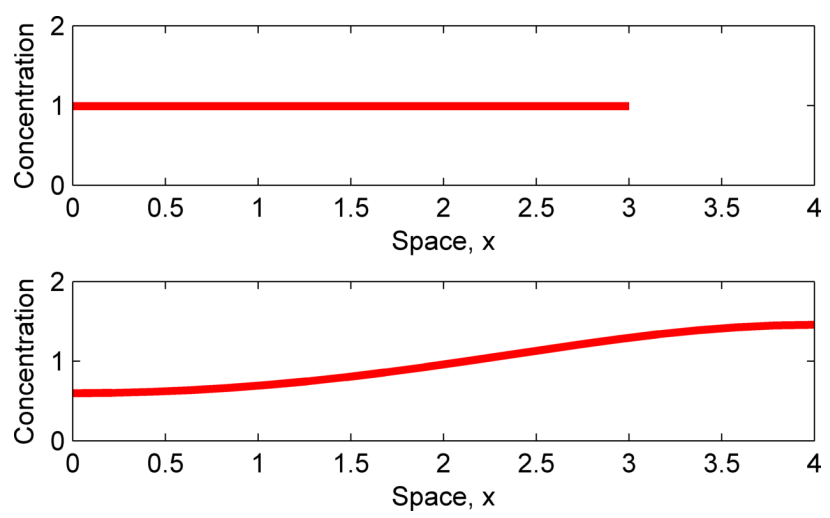

(c)
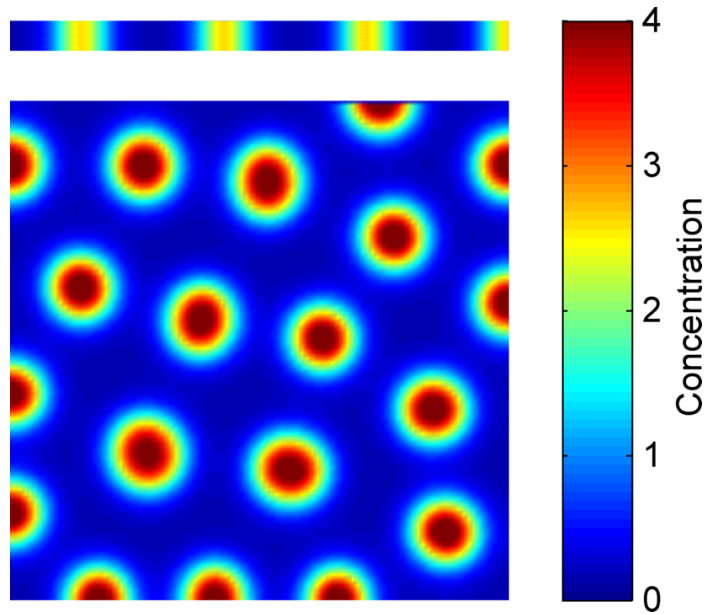

(d)

Fig. 2 Turing pattern properties. (a) Schnakenberg kinetics $D_{1}=1, D_{2}=40, a=0.1$ and $b=0.9$. (b) Gierer-Meinhardt kinetics $D_{1}=0.7, D_{2}=70, a=0.03$ and $b=1$. (c) If the Schnakenberg kinetics are on a domain of length 3 no pattern emerges. However, a domain length of 4 allows heterogeneity to appear. (d) Two-dimensional simulation of the Schnakenberg kinetics. The top simulation shows a thin rectangle that is only able to support stripes across the domain. However, when we increase the vertical height we see that the pattern can produce spots. Note that only one chemical concentration is shown in (c) and (d), the other one will be 180 degrees out of phase. 
Meinhardt, but later derived from a hypothetical chemical reaction using the Law of Mass Action, is the Schnakenberg model [6], which takes the form

$$
f(u, v)=a-u+u^{2} v ; g(u, v)=b-u^{2} v,
$$

where $a$ and $b$ are constants. The Thomas model [7], on the other hand, describes the interaction of uric acid, $u$, with oxygen, $v$, where both reactants diffuse from a reservoir maintained at fixed concentrations, and interact via kinetics empirically determined by data fitting:

$$
f(u, v)=\alpha(a-u)-\frac{u v}{c+u+d u^{2}} ; g(u, v)=\beta(b-v)-\frac{u v}{c+u+d u^{2}},
$$

where $a, b, c, d, \alpha$, and $\beta$ are positive constants. More recently, Barrio et al. [8] proposed a caricature model (which we will denote as BVAM) for ease of analysis. In this model, they simply postulated a system in which the linear, quadratic and cubic terms are explicit:

$$
f(u, v)=a u+v-r_{1} u v^{2}-r_{2} u v ; g(u, v)=b u+c v+r_{1} u v^{2}+r_{2} u v,
$$

where $a, b, c, r_{1}$, and $r_{2}$ are non-negative constants. The inclusion of non-linear terms of this form allowed them to easily consider the case where the linear system is degenerate and the non-linear terms then specify the pattern, with quadratic terms favouring spots and cubic terms favouring stripes [9]. Of course, this model can exhibit negative values of $u$ and $v$ which at first, appear unphysical, but $u$ and $v$ should not be interpreted as concentrations, but rather as deviations from some positive spatially uniform steady state. This seemingly simple model actually gives rise to an incredibly large range of different types of patterns [10]. In Fig. 3, we present a selection of stationary patterns while in Fig. 4 we illustrate some temporally evolving patterns. The latter are non-stationary and their analysis is beyond the scope of this paper.

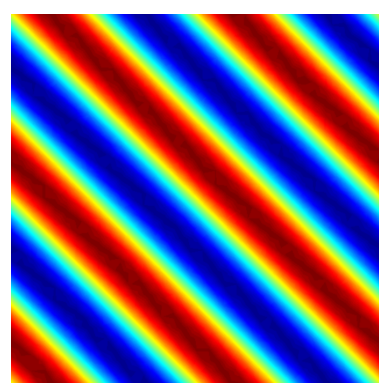

(a)

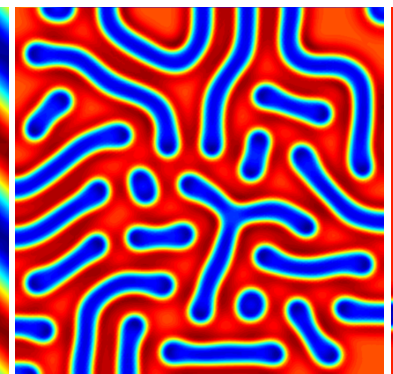

(b)

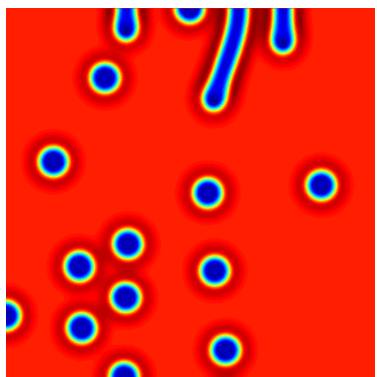

(c)

Fig. 3 Stationary patterns appearing in the BVAM model. We see that we can generate stripes, labyrinthine patterns and spots, respectively. 


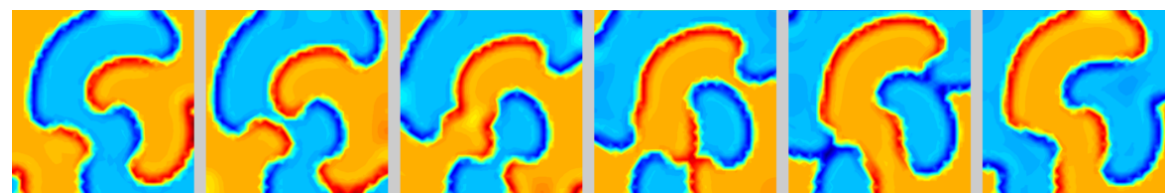

(a)

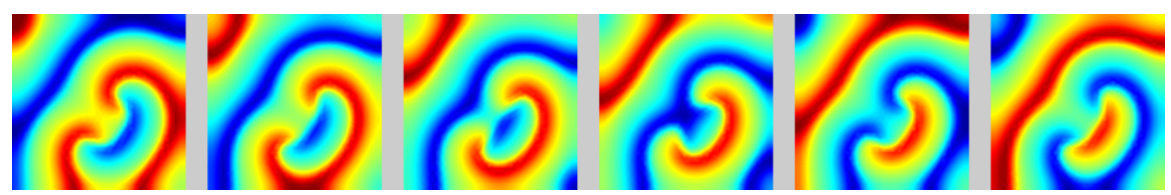

(b)

Fig. 4 Non-stationary patterns appearing in the BVAM model. The images illustrate snapshots of non-stationary patterns, with time increasing to the right along each row. In (a) we see travelling waves, whilst (b) shows scroll waves.

\section{Applications}

The ability of the Turing model to produce patterning has meant that it has been used in a bewildering array of applications, ranging from regeneration in Hydra, to digit patterning, to animal pigmentation, shells, hair, teeth and feather patterns etc. (see [11, 12, 13] and Fig. 5). While the model can produce an astonishing array of patterns, properties 3 and 4 suggest that the patterns that are formed are constricted by domain size. Specifically, property 3 states that if the patterning region is too small then no pattern will form even if the reaction parameters are chosen to produce a Turing pattern. Further, property 4 suggests that as the domain increases in size, the derived linear theory predicts that patterns increase in complexity and, conversely, a decrease in domain dimensions would reduce patterning complexity. This is an example of a developmental constraint [14].

One of the problems with Turing reaction-diffusion models is that the patterns they produce can be very sensitive to small variations in parameter values and to variations in initial conditions, questioning their applicability to situations where robustness is essential - for example, we only want one head! - and this was first pointed out by Bard and Lauder [15]. This sensitivity can either arise due to the fact that the parameter space in which Turing patterns can form can be very small [16] or because the system can exhibit multiple stable spatially heterogeneous solutions (see, for example, Fig. 6).

This issue is still not fully resolved. Dillon et al. [17] showed that choosing different types of boundary conditions could enhance the robustness of some modes while eliminating the admissibility of other modes. Crampin et al. [18] reformulated the model on a growing domain, noting equations of the form (1) will transform to 


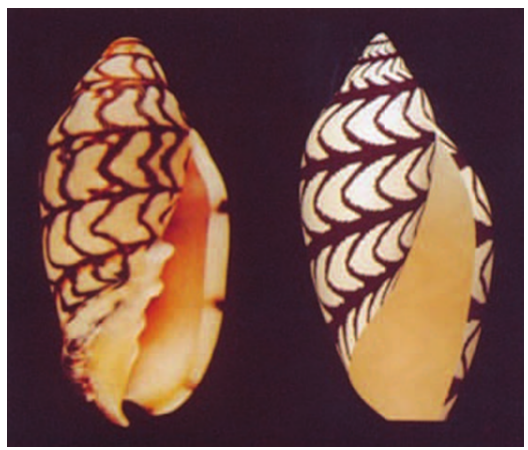

Fig. 5 Illustration of more complex Turing dynamics that can reproduce the patterns seen on seashells. Taken from [12].

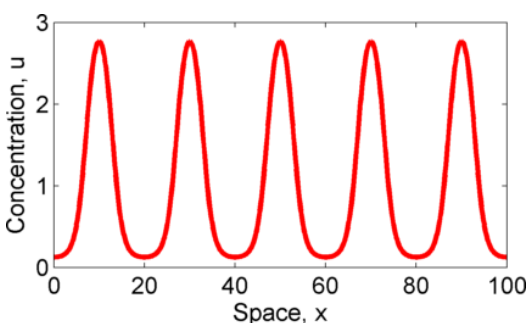

(a)

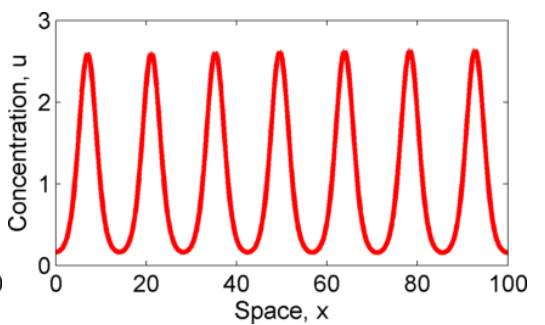

(b)

Fig. 6 Illustrating the robustness issue. All solutions are stationary. The simulations are of the Schnakenberg model [6] and the parameters are the same for both simulations, the only difference being that the initial conditions were two different randomly chosen states which were uniformly distributed around their unique spatially uniform steady state.

$$
\frac{\partial u}{\partial t}+\frac{\partial s u}{\partial x}=D \frac{\partial^{2} u}{\partial x^{2}}+f(u)
$$

where $s$ is the velocity of flow induced by growth (in higher dimensions, this term would take the form $\nabla .(s u)$, see the original paper for the derivation of this form). They showed that this system could robustly generate mode doubling for the case of uniform domain growth as well as generating, in a robust fashion, a sequence of consecutive modes for the case of apical growth (Fig. 7).

An obvious question to ask now is, if pattern complexity increases with domain size under this theory, then shouldn't we have a large number of heads? However, cells can only respond to signals for a certain time window before they differentiate and therefore can no longer respond to changing signals. One dramatic situation in which this is not the case is pigmentation patterning in certain fishes where, as the domain grows, the pattern continually changes to preserve wavelength, again consistent with the Turing model [19]. 


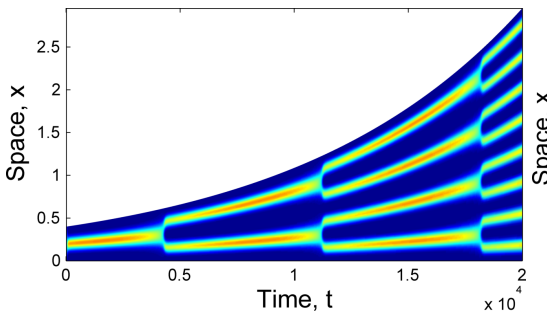

(a)

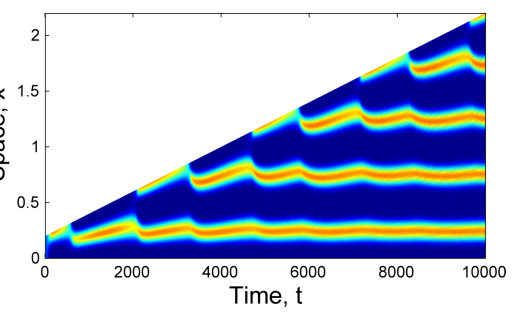

(b)

Fig. 7 Deterministic simulations of the Schnakenberg model [6] on a domain growing (a) exponentially uniformly everywhere, and (b) linearly, but only at the tip (apical growth).

Another key issue is the identification of the morphogens involved. While this remains a controversial issue, there have been many potential activator-inhibitor pairs identified (see, for example [20, 21, 22]). Moreover, it has been posited that the activator-inhibitor system might actually be composed of cells - a particular example being the interaction of melanophores and xanthophores in zebrafish pigementation patterning $[23,24,25]$.

While in the above we have considered robustness in response to different initial conditions or parameter values, none of these studies investigated the effect of noise, which we would expect to be present in a biological system throughout the patterning process. Woolley et al. [26] showed the presence of noise could disrupt the robust period-doubling patterning sequence seen by Crampin et al. [18] (Fig. 7) but robustness was preserved in the case of apical growth (Fig. 8).

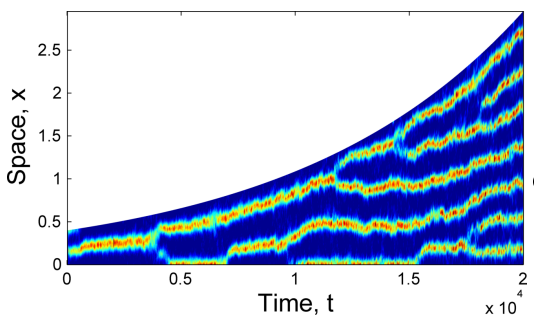

(a)

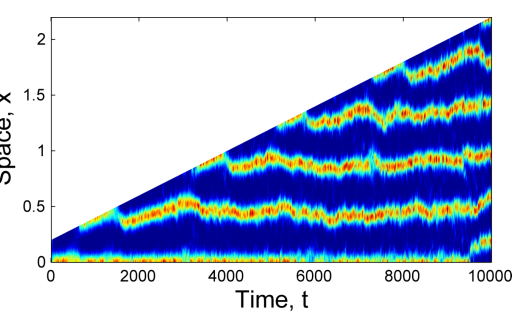

(b)

Fig. 8 Stochastic simulations of the Schnakenberg model [6] on a domain growing (a) exponentially uniformly everywhere, and (b) linearly, but only at the tip (apical growth).

This offers a possible reason why, in biology, we usually see patterns forming behind a propagating front, rather than simultaneously across the full domain. A propagating front allows patterns to form in a sequentially controlled and robust fashion. 
The robustness issue has recently been tackled in a different way by Kurics et al. [27] who showed that extending the Turing model to be more biologically realistic by including receptor and feedback dynamics actually could greatly enhance the parameter space in which patterns are predicted.

While still controversial biologically, Turing structures have been found in chemistry - the first example being the Chloride-Iodide-Malonic-Acid (or CIMA) reaction [28]. One of the reasons why it had been difficult to find Turing structures in chemistry was due to property 1 in Sect. 2.2, namely that the diffusion coefficients $D_{1}$ and $D_{2}$, must be different. While it is possible, theoretically, to obtain Turing structures for $D_{1}$ and $D_{2}$ arbitrarily close to one another [29], for robust patterning $D_{1}$ and $D_{2}$ have to be quite different from each other and, typically, when chemicals react with each other the chemical molecules have similar sizes and therefore quite similar diffusion coefficients. In the CIMA reaction, however, one of the reactants was bound to starch as an indicator and this changed its diffusion coefficient significantly to move the system into the Turing patterning regime. This was modelled by Lengyel and Epstein [30] in the following way:

$$
\begin{aligned}
& \frac{\partial u}{\partial t}=D_{1} \frac{\partial^{2} u}{\partial x^{2}}+f(u, v)-c_{0} u p_{+}+c p_{-} \\
& \frac{\partial v}{\partial t}=D_{2} \frac{\partial^{2} v}{\partial x^{2}}+g(u, v) \\
& \frac{\partial c}{\partial t}=c_{0} u p_{+}-c p_{-} .
\end{aligned}
$$

Here, $u$ and $v$ are the concentrations of the chemical species and $u$ is assumed to be interacting with the indicator (starch). Assuming that the starch is in excess we can take its concentration to be fixed at $c_{0}$. Then, by the Law of Mass Action, the rate at which $u$ binds with starch to create the complex $c$ is $c_{0} u p_{+}$, where $p_{+}$is a rate constant, while the rate at which $u$ is recovered from the complex is $c p_{-}$. Assuming the indicator, and therefore the complex, is immobile, we obtain the equation for the complex $c$.

Adding equations (25) and (27) we obtain

$$
\frac{\partial(u+c)}{\partial t}=D_{1} \frac{\partial^{2} u}{\partial x^{2}}+f(u, v) .
$$

The further assumption that the binding between $u$ and the indicator is fast, allows us to replace $c$ in the equation (27) by $P u$, where $P=c_{0} p_{+} / p_{-}$, which essentially rescales the diffusion coefficient $D_{1}$ by a factor $1 /(1+P)$ (recall that for diffusiondriven instability $D_{1}<D_{2}$ assuming $u$ is the activator and $v$ the inhibitor. Hence, $D_{1}$ is effectively lowered).

Turing's original derivation of his reaction-diffusion model was on a discrete array of "cells" or compartments in which reactions took place while the chemicals were transported down chemical gradients to neighbouring compartments. $\mathrm{He}$ essentially arrived at a spatially discretised version of the system described in Sect. 2.2. Recently, Tompkins et al. [31] actually made a physical model of this 
set up with compartments in which chemicals reacted and diffused to neighbouring compartments. They showed that this system could produce patterns.

\section{Conclusions and Discussion}

We have shown how spatial patterning can arise from a coupled system of two reaction-diffusion equations and given some examples of applications of the theory of diffusion-driven instability in biology. We have only looked at the basic Turing model but, since Turing's original paper, there have been many extensions made of the model. For example, in a series of papers Nagorcka and colleagues [32,33] proposed that the initial structures formed by a Turing model could serve as sources or sinks of further Turing models, leading to very complex patterned structures similar to those observed in hair follicles and feather primorida. Kondo and colleagues have carried out extensive experimental studies on fish pattern regeneration, patterns on fish mutants and addressed the issue of how one could link the parameters in a Turing model with more refined genetic information (see the review: [34]).

It is important to point out that there are many other self-organisation models that can produce patterns. For example, in 1983, Oster, Murray and Harris [35, 36] proposed that patterns arose due to cells mechanically interacting with each other, leading to spatially heterogeneous patterns of cells themselves, which they then assumed differentiated into structures. It is also known that cells can move in response to gradients in chemicals (chemotaxis) and it has been shown that such chemotaxis models can also lead to spatial pattern formation Keller and Segel [37]. Painter et al., $[38,39]$ showed how a Turing system combined with chemotaxis could lead to patterns of varying wavelengths, consistent with those formed in Pomacanthus and generalising the concept of positional information [40].

In summary the Turing model has generated a great deal of experimental and mathematical interest, which continues to this day.

\section{Acknowledgements}

PKM and TEW would like to thank the Mathematical Biosciences Institute (MBI) at Ohio State University, for financially supporting this research through the National Science Foundation grant DMS 1440386. TEW would also like to acknowledge financial support from St John's College, Oxford and BBSRC grant BKNXBKOO BK00.16.

The zebra skin, serval, arrow frog and x-ray pictures from Figure 1 are used under creative commons license 2.0. They were downloaded from flickr.com and owned by David Richards, Vince Smith, TheReptillarium and Rahim Packir Saibo, respectively. 


\section{References}

[1] A. M. Turing. The chemical basis of morphogenesis. Phil. Trans. R. Soc. Lond. B, 237:37-72, 1952.

[2] T. T. Marquez-Lago and P. Padilla. A selection criterion for patterns in reaction-diffusion systems. Theor. Biol. Med. Modell., 11(1):7, 2014.

[3] P. Grindrod. The Theory and Applications of Reaction-Diffusion Equations: Patterns and Waves. Clarendon Press, 1996.

[4] N. F. Britton. Reaction-diffusion Equations and Their Applications to Biology. Academic Press, London, 1986.

[5] A. Gierer and H. Meinhardt. A theory of biological pattern formation. Biol. Cybern., 12(1):30-39, 1972.

[6] J. Schnakenberg. Simple chemical reaction systems with limit cycle behaviour. J. Theor. Biol., 81(3):389-400, 1979.

[7] D. Thomas. Analysis and Control of Immobilised Enzyme Systems, chapter Artificial enzyme membranes, transport, memory, and oscillatory phenomena, pages 115-150. Berlin, Heidelberg, New York, Springer, 1975.

[8] R. A. Barrio, C. Varea, J. L. Aragón, and P. K. Maini. A two-dimensional numerical study of spatial pattern formation in interacting Turing systems. Bull. Math. Biol., 61(3):483-505, 1999.

[9] B. Ermentrout. Stripes or spots? Nonlinear effects in bifurcation of reactiondiffusion equations on the square. Proc. Math. Phys. Sci., 434(1891):413-417, 1991.

[10] T. E. Woolley, R. E. Baker, P. K. Maini, J. L. Aragón, and R. A. Barrio. Analysis of stationary droplets in a generic Turing reaction-diffusion system. Phys. Rev. E, 82(5):051929, 2010.

[11] H. Meinhardt. Models of Biological Pattern Formation. Academic Press, 1982.

[12] H. Meinhardt. The Algorithmic Beauty of Sea Shells. Springer Verlag, 2009.

[13] J. D. Murray. Mathematical Biology II: Spatial Models and Biomedical Applications, volume 2. Springer-Verlag, 3rd edition, 2003.

[14] G. F. Oster, N. Shubin, J. D. Murray, and P. Alberch. Evolution and morphogenetic rules: the shape of the vertebrate limb in ontogeny and phylogeny. Evolution, pages 862-884, 1988.

[15] J. Bard and I. Lauder. How well does Turing's theory of morphogenesis work? J. Theor. Biol., 45(2):501-31, 1974.

[16] J. D. Murray. Parameter space for Turing instability in reaction diffusion mechanisms: a comparison of models. J. Theor. Biol., 98(1):143, 1982.

[17] R. Dillon, P. K. Maini, and H. G. Othmer. Pattern formation in generalized Turing systems. J. Math. Biol., 32(4):345-393, 1994.

[18] E. J. Crampin, E. A. Gaffney, and P. K. Maini. Reaction and diffusion on growing domains: scenarios for robust pattern formation. Bull. Math. Biol., 61 (6):1093-1120, 1999.

[19] S. Kondo and R. Asai. A reaction-diffusion wave on the skin of the marine angelfish Pomacanthus. Nature, 376:765-768, 1995. 
[20] S. A. Newman and R. Bhat. Activator-inhibitor dynamics of vertebrate limb pattern formation. Birth Defects Res. C Embryo Today, 81(4):305-319, 2007.

[21] S. Sick, S. Reinker, J. Timmer, and T. Schlake. WNT and DKK determine hair follicle spacing through a reaction-diffusion mechanism. Science, 314(5804): 1447-1450, 2006.

[22] A. Garfinkel, Y. Tintut, D. Petrasek, K. Boström, and L. L. Demer. Pattern formation by vascular mesenchymal cells. Proc. Nat. Acad. Sci., 101(25): 9247, 2004.

[23] A. Nakamasu, G. Takahashi, A. Kanbe, and S. Kondo. Interactions between zebrafish pigment cells responsible for the generation of Turing patterns. Proc. Nat. Acad. Sci., 106(21):8429-8434, 2009.

[24] T. E. Woolley. Pattern production through a chiral chasing mechanism. Phys. Rev. E, 96(3):032401, 2017.

[25] T. E. Woolley, P. K. Maini, and E. A. Gaffney. Is pigment cell pattern formation in zebrafish a game of cops and robbers? Pig. Cell Melan. Res., 27(5):686687, 2014.

[26] T. E. Woolley, R. E. Baker, E. A. Gaffney, and P. K. Maini. Stochastic reaction and diffusion on growing domains: Understanding the breakdown of robust pattern formation. Phys. Rev. E, 84(4):046216, 2011.

[27] T. Kurics, D. Menshykau, and D. Iber. Feedback, receptor clustering, and receptor restriction to single cells yield large Turing spaces for ligand-receptorbased Turing models. Phys. Rev. E, 90(2):022716, 2014.

[28] V. Castets, E. Dulos, J. Boissonade, and P. De Kepper. Experimental evidence of a sustained standing Turing-type nonequilibrium chemical pattern. Phys. Rev. Lett., 64(24):2953-2956, 1990.

[29] J. E. Pearson and W. Horsthemke. Turing instabilities with nearly equal diffusion coefficients. J. Chem. Phys., 90:1588, 1989.

[30] I Lengyel and I. R. Epstein. A chemical approach to designing Turing patterns in reaction-diffusion systems. Proc. Nat. Acad. Sci., 89(9):3977-3979, 1992.

[31] N. Tompkins, N. Li, C. Girabawe, M. Heymann, G. B. Ermentrout, I. R. Epstein, and S Fraden. Testing Turings theory of morphogenesis in chemical cells. Proc. Nat. Acad. Sci., 2014.

[32] B. N. Nagorcka. Wavelike isomorphic prepatterns in development. J Theor. Biol., 137(2):127-162, 1989.

[33] J. R. Mooney and B. N. Nagorcka. Spatial patterns produced by a reactiondiffusion system in primary hair follicles. J. Theor. Biol., 115(2):299-317, 1985.

[34] S. Kondo, M. Iwashita, and M. Yamaguchi. How animals get their skin patterns: fish pigment pattern as a live Turing wave. Int. J. Dev. Biol., 53(5-6): $851,2009$.

[35] G. F. Oster, J. D. Murray, and A. K. Harris. Mechanical aspects of mesenchymal morphogenesis. Development, 78(1):83-125, 1983.

[36] J. D. Murray, G. F. Oster, and A. K. Harris. A mechanical model for mesenchymal morphogenesis. J. Math. Biol., 17(1):125-129, 1983. 
[37] E. F. Keller and L. A. Segel. Initiation of slime mold aggregation viewed as an instability. J. Theor. Biol., 26(3):399-415, 1970.

[38] K. J. Painter, P. K. Maini, and H. G. Othmer. Stripe formation in juvenile Pomacanthus explained by a generalized Turing mechanism with chemotaxis. Proc. Nat. Acad. Sci., 96(10):5549, 1999.

[39] K. J. Painter, P. K. Maini, and H. G. Othmer. Development and applications of a model for cellular response to multiple chemotactic cues. J. Math. Biol., 41 (4):285-314, 2000.

[40] L. Wolpert. Positional information and pattern formation. Curr. Top. Dev. Biol., 6:183-224, 1971. 